\title{
Influencia del arenado de la aleación Ti6Al4V en la dureza subsuperficial y estado de tensiones residuales ${ }^{(\cdot)}$
}

\author{
M. Multigner ${ }^{*, * *}$, P. Fernández-Castrillo*, S. Ferreira-Barragans*, G. González-Doncel ${ }^{*}$ y J. L. González-Carrasco,** \\ Resumen \\ El arenado de la aleación Ti6Al4V es un tratamiento orientado a la mejora de la fijación mecánica de los implantes. \\ Sin embargo, dicho tratamiento contribuye a una disminución de la resistencia a la fatiga del material. En este tra- \\ bajo se ha determinado, mediante radiación sincrotrón, el estado de tensiones residuales en muestras arenadas de es- \\ ta aleación y su posterior proceso de relajación tras un recocido a $500^{\circ} \mathrm{C}$. Se observa que el arenado ocasiona un \\ gradiente de tensiones residuales de compresión con valores de hasta unos $700 \mathrm{MPa}$ en las proximidades de la super- \\ ficie. A pesar de la deformación severa que experimenta el material en esta zona, las medidas de microdureza arrojan \\ valores similares a los obtenidos en el interior del material. Tras el tratamiento térmico se observa una relajación de \\ las tensiones residuales y un importante aumento de la dureza superficial.
}

Palabras clave Ti6Al4V; Arenado; Tensiones residuales; Radiación sincrotrón; Biomateriales.

\section{Influence of sandblasting of Ti6Al4V alloy on the subsurface hardness and residual stresses state}

\begin{abstract}
Sandblasting of Ti6Al4V alloy is used to enhance mechanical fixation of the implants. Under the mechanical point of view, such treatment yields a decrease of the fatigue resistance. In this work we evaluate by means of synchrotron radiation the residual stress state in sandblasted specimens and the further relaxation process after a thermal oxidation treatment at $500{ }^{\circ} \mathrm{C}$. It has been found a significant compressive stress gradient with a maximum of about $700 \mathrm{MPa}$ near the surface. Despite the severe deformation experienced by the surface during sandblasting microhardness measurements failed to show any sufsurface hardening. A thermal oxidation treatment $\left(500^{\circ} \mathrm{C} / 1 \mathrm{~h}\right)$ of the sandblasted specimen yield the stress relief and a significant subsurface hardening.
\end{abstract}

Keywords

Ti6A14V; Sandblasting; Residual stresses; Synchrotron radiation; Biomaterials.

\section{INTRODUCCIÓN}

La aleación Ti6Al4V combina buenas propiedades mecánicas y una buena biocompatibilidad tanto in vitro como in vivo, por lo que es muy empleada en la fabricación de una gran variedad de dispositivos para aplicaciones biomédicas. Teniendo en cuenta que la respuesta biológica está estrechamente relacionada con las propiedades superficiales, es comprensible que una de las actividades más importantes en el sector de implantes haya estado encaminada al uso de recubrimientos (orgánicos e inorgánicos) o a modificaciones superficiales mediante métodos físicos o químicos que no comprometan las propiedades mecánicas del sustrato. Uno de los métodos más utilizados para mejorar la osteointegración del implante consiste en un tratamiento de arenado con partículas de alúmina o silicatos cuyo tamaño, forma y energía cinética condicionan fuertemente la rugosidad. El arenado cambia la composición de la superficie ${ }^{[1-3]}$, aunque la presencia de las partículas residuales no parece tener un efecto en la osteointegración ${ }^{[4]}$. Datos obtenidos in vivo han puesto de manifiesto que las superficies rugosas producen una fijación mecánica u osteointegración mejor que la correspondiente a superficies lisas ${ }^{[5-7]}$. Este tratamiento tampoco compromete el buen comportamiento a corrosión in vitro de las aleaciones de titanio $^{[3 \text { y } 8]}$.

Los estudios realizados por otros autores sobre el efecto de dicho tratamiento superficial en la aleación Ti6Al4V han puesto de manifiesto una pérdida

\footnotetext{
(•) Trabajo recibido el día 15 de enero de 2008 y aceptado en su forma final el día 16 de junio de 2008.

* Centro Nacional de Investigaciones Metalurgicas (CENIM-CSIC).

** Centro de Investigación Biomédica en Red en Bioingeniería, Biomateriales y Nanomedicina (CIBER-BBN), Madrid, España.
} 
importante de la resistencia a la fatiga ${ }^{[9]}$, lo que contrasta con los resultados publicados para titanio puro $^{[8 \text { y } 10]}$. La resistencia a la fatiga disminuye con el incremento de la rugosidad: un $20 \%$ en las probetas con un arenado fino y hasta un $40 \%$ en las probetas con un arenado basto ${ }^{[11]}$.

La deformación plástica severa asociada al proceso de arenado da lugar al desarrollo de tensiones de compresión superficiales. En el caso de titanio puro se han reportado valores de aproximadamente 480 $\mathrm{MPa}^{[8]}$. Para la aleación Ti6Al4V, Leinenbach y Eifler $^{[9]}$ demostraron que las tensiones residuales causadas por el desbaste y pulido disminuyen de 400 a $160 \mathrm{MPa}$ por el proceso de granallado. Sin embargo, Martínez et al. ${ }^{[12]}$ encontraron valores de aproximadamente $895 \mathrm{MPa}$ después del shot peening.

Estudios recientes con cultivos de osteoblastos primarios han demostrado que un tratamiento de oxidación $\left(500^{\circ} \mathrm{C} / 1 \mathrm{~h}\right)$ de las aleaciones chorreadas con alúmina mejora la adhesión celular, proliferación y organización del citoesqueleto respecto a las probetas arenadas no tratadas. No se dispone de información relativa al efecto de dicho tratamiento en el estado de tensiones residuales y propiedades mecánicas subsuperficiales.

El objetivo de este trabajo es investigar el efecto del tratamiento de arenado y posterior tratamiento térmico de oxidación en las tensiones residuales en la zona afectada por el arenado y zonas adyacentes y en las propiedades mecánicas subsuperficiales. El estudio está encaminado a comprender y explotar las propiedades superficiales que, además de influir en la respuesta biológica, pueden ser cruciales en componentes sometidos a carga donde la resistencia a la fatiga suele ser el principal requisito para alcanzar una determinada biofuncionalidad.

\section{MATERIALESY MÉTODOS}

Se han utilizado discos de Ti6Al4V de $20 \mathrm{~mm}$ de diámetro y $2 \mathrm{~mm}$ de espesor suministrados por BIOMET (Valencia, España). Las superficies investigadas han sido pulidas, arenadas, además de arenadas y oxidadas térmicamente. Las probetas pulidas (Ti64) se prepararon mediante desbaste con lijas de $\mathrm{SiC}$, sucesivamente más finas, y pulido mecánico con pasta de diamante. Por último, las probetas se pulieron utilizando sílice coloidal. Las superficies arenadas (BL1), fueron preparadas por el fabricante aplicando un chorro de partículas de alúmina de $~ 500 \mu \mathrm{m}$ de diámetro, utilizando una presión de $60 \pm 10$ psi, a una distancia de unos $80 \mathrm{~mm}$ de la boquilla, durante unos pocos minutos. Después del arenado, las probetas se sometieron a un lavado con agua que contenía detergente a $75^{\circ} \mathrm{C}$ durante $5 \mathrm{~min}$, seguido de un ataque ácido en una solución de $\mathrm{HNO}_{3}$ a, aproximadamente, $50{ }^{\circ} \mathrm{C}$ durante $15 \mathrm{~min}$. Finalmente, las probetas se lavaron con agua ultra pura durante $3 \mathrm{~min}$. Antes de su uso, ambos tipos de muestras fueron sonicadas en alcohol. La tercera condición superficial (BL1 - 500) se preparó mediante un tratamiento a $500^{\circ} \mathrm{C}$, en aire, de las probetas arenadas y se enfriaron hasta alcanzar la temperatura ambiente fuera del horno. Con fines comparativos, se ha estudiado también un juego de probetas (BL2) preparadas por SURGIVAL (Valencia, España), utilizando para el arenado partículas de alúmina de unas $750 \mu \mathrm{m}$ de diámetro y una presión de 50,7 psi.

La caracterización microestructural se realizó mediante microscopia electrónica de barrido (MEB), utilizando un microscopio electrónico (JEOL-6500F) y un sistema de energías dispersivas de rayos $\mathrm{X}$, acoplado al microscopio, para el microanálisis químico. La rugosidad se determinó en un profilómetro Mitotuyo Surftest 401, antes y después de la modificación superficial. Las medidas se obtuvieron a partir de perfiles de línea con un barrido de $4 \mathrm{~mm}$. Se determinó el valor medio de la rugosidad $\left(\mathrm{R}_{\mathrm{a}}\right)$ con una sensibilidad de $0,01 \mu \mathrm{m}$.

Las medidas de tensiones residuales se determinaron mediante difracción de radiación sincrotrón en el instrumento EDDI (BESSY, Berlin, Alemania), por el método de dispersión de energías ${ }^{[13]}$. Se empleó radiación con energías entre 10 y $150 \mathrm{keV}$. La principal diferencia en relación con el análisis mediante difracción de rayos X convencional es que la radiación sincrotrón es radiación $X$ de alta energía, por lo que posee mayor poder de penetración permitiendo, además, la determinación de tensiones internas en volúmenes sensiblemente más pequeños. Se ha investigado el perfil de las tensiones residuales en una región adyacente a la superficie, con una profundidad de hasta unas $50 \mu \mathrm{m}$, utilizando el método puesto a punto por Genzel et al. ${ }^{[14]}$. Mediante éste método, es posible determinar, para medidas realizadas a diferentes ángulos de la muestra en un plano perpendicular al plano de difracción, las tensiones a distintas profundidades a partir de la información suministrada por los diferentes picos de difracción. El ángulo de difracción (20) utilizado ha sido $6^{\circ}$ y las reflexiones analizadas las (100), (002), (101) y (102) correspondientes a la fase $\beta$. Con fines comparativos, se determinó el estado de tensiones residuales en muestras pulidas.

Para analizar el efecto del arenado en la dureza del material se realizaron medidas de dureza Vickers sobre secciones transversales pulidas. Para preservar la zona superficial durante la preparación de la sección, las probetas fueron recubiertas antes del corte 
con un recubrimiento electrolítico de cobre. Las medidas se realizaron en un microdurómetro Wilson utilizando cargas de $10 \mathrm{~g}$ y tiempos de indentación de $15 \mathrm{~s}$. Se han realizado perfiles de dureza en profundidad, desde la superficie arenada y a lo largo de una banda paralela a la interfaz cobre/sustrato. Cada valor corresponde a la media de 10 indentaciones.

\section{RESULTADOSY DISCUSIÓN}

\subsection{Caracterización del material}

El análisis mediante MEB de la probeta pulida revela una estructura bifásica consistente en zonas claras (fase $\beta$ ) dentro de una matriz oscura (fase $\alpha$ ) (Fig.1). El arenado de la aleación con partículas de alúmina modifica las superficies en dos aspectos: a) La rugosidad $\left(R_{a}\right)$ aumenta desde 0,07 $\mu \mathrm{m}$ en la condición de pulido hasta $2 \mu \mathrm{m}$ después del arenado; b) La superficie está severamente deformada, con numerosas indentaciones irregulares y bordes muy acusados. Una observación a más aumentos de la superficie revela una distribución heterogénea de partículas oscuras, a menudo fracturadas, con bordes poligonales, $y$ un tamaño medio de, aproximadamente, $20 \mu \mathrm{m}$ (Fig. 2 a)). Algunos de los fragmentos de las partículas de mayor tamaño permanecen firmemente adheridos a la superficie. En el caso de la probeta facilitada por Surgival (Fig. 2 b)) se aprecia una superficie menos rugosa, con un $R_{a}$ de 0,55 $\pm 0,05 \mu \mathrm{m}$. En ambas probetas se aprecian pequeñas grietas superficiales. $\mathrm{La}$

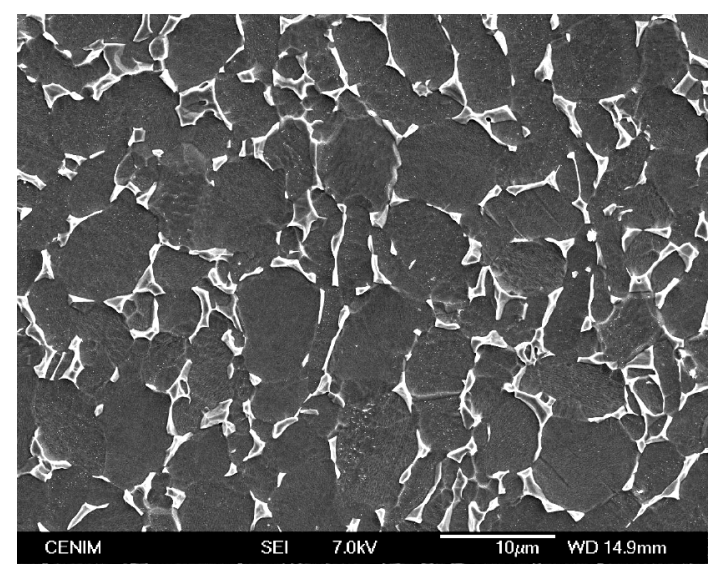

Figura 1. Imagen de electrones secundarios que revela la microestructura bifásica de la aleación Ti6Al4V

Figure 1. Backscattered electron images revealing the biphasic miscrostructure of the Ti6Al4V alloy.
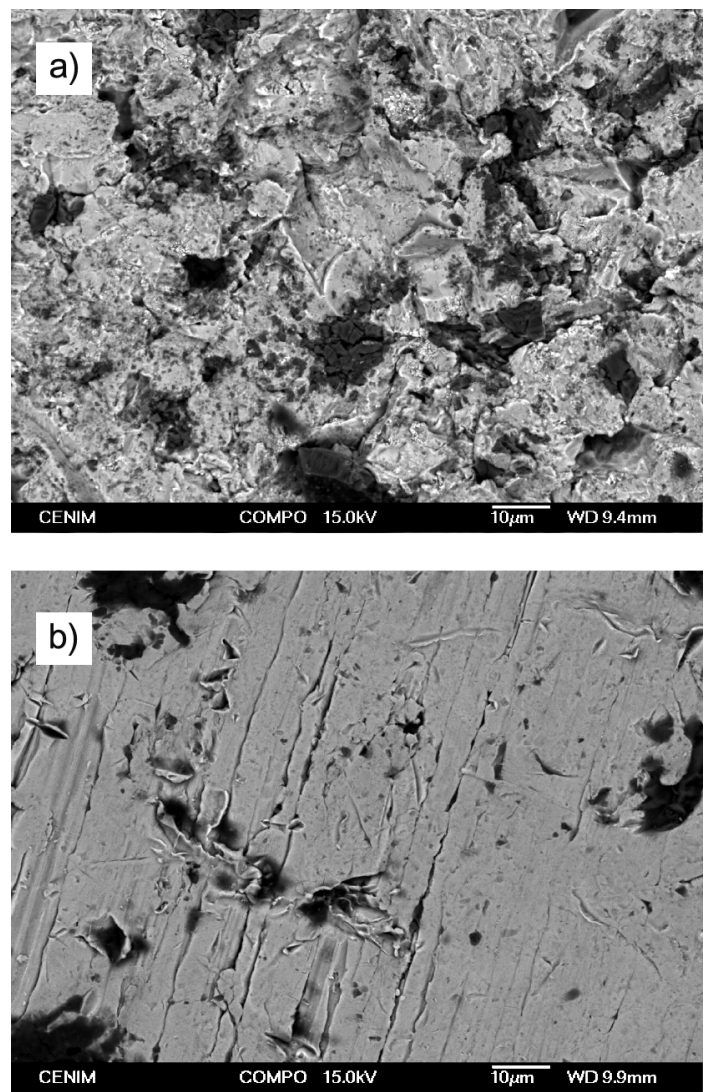

Figura 2. Imágenes de electrones retrodispersados de la superficie de las probetas arenadas. a) BL1 y b) BL2.

Figure 2. Backscattered electron images of the surface of sandblasted specimens. a) BL 1 and b) $B L 2$.

formación de grietas durante el arenado se ha observado en probetas de Ti6Al4V pero no en titanio puro dada su mayor ductilidad ${ }^{[15]}$.

La oxidación térmica produce un cambio en la coloración superficial que evoluciona desde el gris metálico, para la superficie arenada, hasta un azulado en la probeta oxidada. Dicha coloración se debe al aumento de espesor de la capa de pasivado que recubre la aleación ${ }^{[16]}$. Teniendo en cuenta la correlación color-espesor que establecen los autores de dicho trabajo puede estimarse un espesor de la capa de óxido de unos $100 \mathrm{~nm}$. La rugosidad de la probeta arenada oxidada (BL1-500) es 1,6 $\pm 0,5 \mu \mathrm{m}$, que es ligeramente inferior al valor de $2 \pm 0,4 \mu \mathrm{m}$ determinado para la probeta no oxidada (BL1). Dados los intervalos de desviación estándar de los valores medios puede afirmarse que la diferencia no es significativa.

El análisis de las secciones transversales pone de manifiesto la presencia de fragmentos de partículas de alúmina incrustadas en la superficie, que se han 
INFLUENCIA DEL ARENADO DE LA ALEACIÓN TI6AL4V EN LA DUREZA SUBSUPERFICIAL Y ESTADO DE TENSIONES RESIDUALES INFLUENCE OF SANDBLASTING OF TI6AL4V ALLOY ON THE SUBSURFACE HARDNESS AND RESIDUAL STRESSES STATE

generado durante el proceso de arenado (Fig. 3). Una observación detallada pone de manifiesto la ausencia de grietas, lo que confirma la escasa profundidad de las observadas durante el examen de la superficie. El cambio de la morfología de la fase $\beta$ en las inmediaciones de la superficie revela una banda, de unas 5 $\mu \mathrm{m}$ de espesor, que parece estar afectada por la deformación severa de la superficie.

\subsection{Estudio de tensiones residuales}

La figura 4 muestra la evolución de la tensión residual en el plano $\left(\sigma_{22} \text { y } \sigma_{33}\right)^{[17]}$ con la distancia a la superficie para las probetas pulidas, las arenadas y las arenadas y oxidadas. Las probetas pulidas muestran tensiones residuales de compresión de pequeña magnitud en las proximidades de la superficie. Su origen es incierto. No obstante, dada la baja conductividad térmica del titanio podrían estar relacionadas con las operaciones de corte de los discos o con un exceso de presión durante las operaciones de desbaste y pulido.

En las probetas BL1 se pone de manifiesto el notable incremento de las tensiónes residuales. Dichas tensiones son de compresión y muestran un perfil en el que la tensión máxima está cerca de la superficie. La tensión decae a medida que nos alejamos de la misma. La magnitud de dicha tensión es todavía importante a una profundidad de $50 \mu \mathrm{m}$, que es la máxima a la que se ha realizado el estudio. Los valores de tensión obtenidos para las probetas BL2 son inferiores a los medidos para la probeta BL1. Ello podría es-

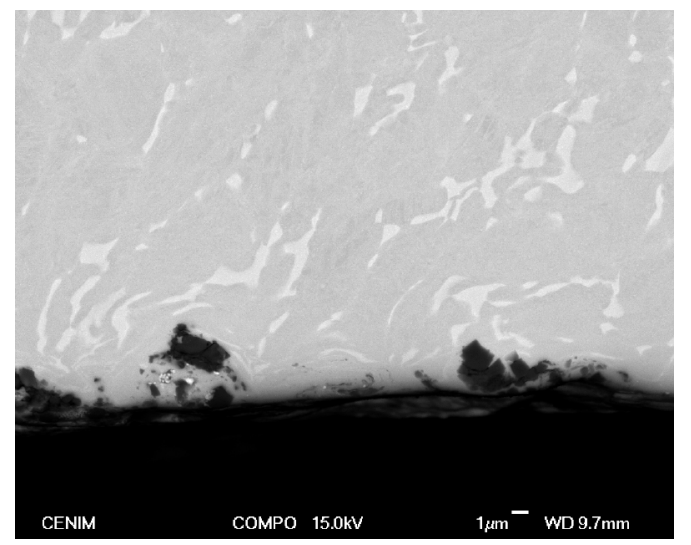

Figura 3. Imagen de electrones retrodispersados correspondientes a una sección transversal de la probeta BL1.

Figure 3. Backscattered electron image corresponding to a transverse section of the BL1 specimen.

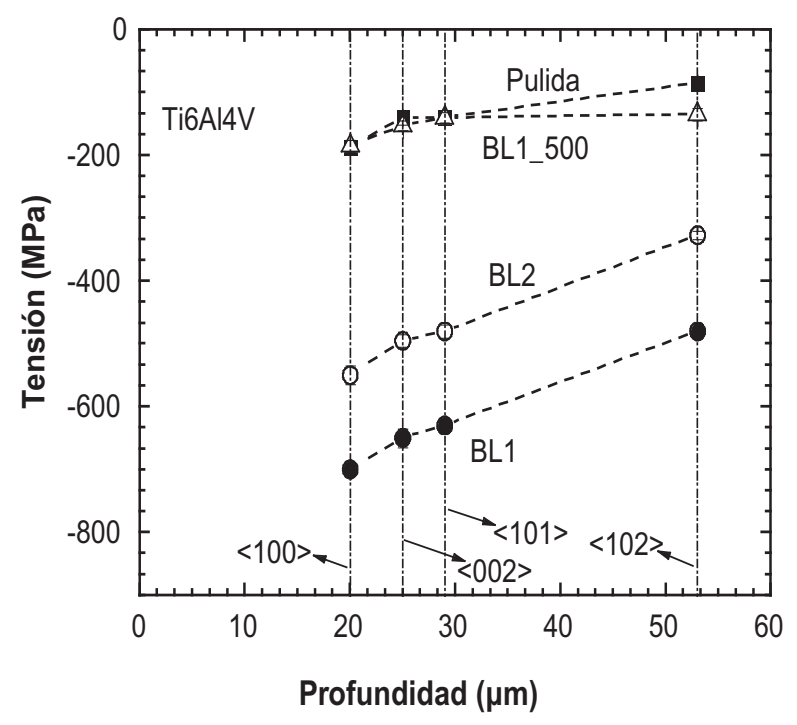

Figura 4. Variación de la tensión residual en función de la distancia a la interfaz.

Figure 4. Variation of the residual stresses as a function of the distance to the surface.

tar en concordancia con su menor rugosidad (Fig. 2 b)), lo que se asocia a una deformación de la superficie menos severa.

El tratamiento de oxidación a $500^{\circ} \mathrm{C}$ de las probetas arenadas relaja significativamente dichas tensiones si bien no las anula. Las tensiones residuales después de la oxidación podrían estar relacionadas con la disolución de oxígeno en las proximidades de la superficie.

\subsection{Estudios de microdureza}

Los resultados obtenidos a lo largo de líneas perpendiculares a la superficie arenada (Fig. 5) ponen de manifiesto una gran dispersión de las medidas de microdureza a lo largo de la línea y de una línea a otra, no pudiendo establecerse una tendencia clara de la variación en dureza con la profundidad. Dicha dispersión podría estar relacionada con el pequeño tamaño de las huellas, con diagonales entre 6 y $8 \mu \mathrm{m}$, que son del orden de magnitud del tamaño de la fase $\beta$ $(\sim 1 \mu \mathrm{m})$. Para minimizar este efecto y poder obtener un valor medio que tuviera significado estadístico, se realizaron varias medidas de microdureza a lo largo de una banda paralela a la superficie situada a unas $20 \mu \mathrm{m}$ de la superficie arenada y en otra situada en el centro de la probeta, que se considera no está afectada por el tratamiento de arenado. En la figura 6 se resumen dichos resultados. Se observa que la microdureza en las proximidades de la superficie de la 


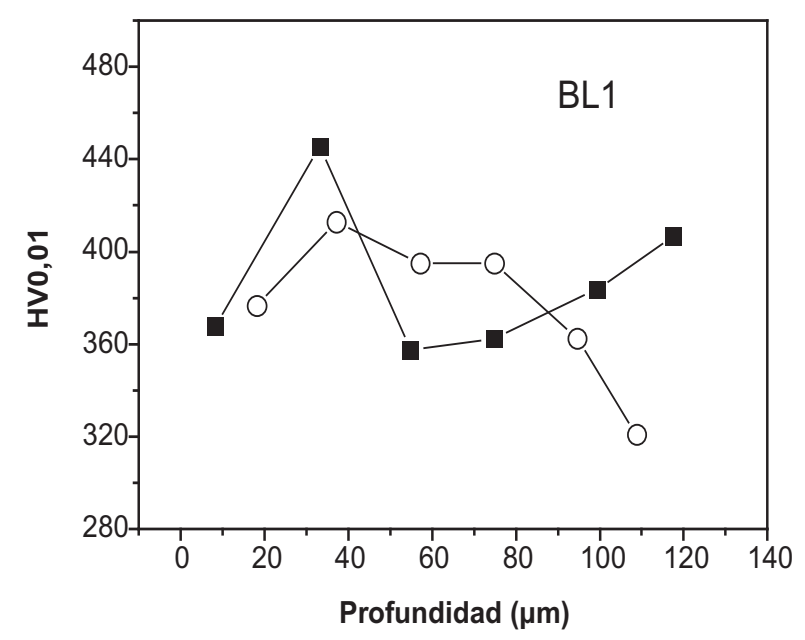

Figura 5. Microdureza en función de la profundidad para la probeta BL1. Cada curva representa una línea de medidas perpendicular a la superficie.

Figure 5. Microhardness values as a function of depth for the BL 1 specimen. Each curve represents a line of measurements perpendicular to the surface.

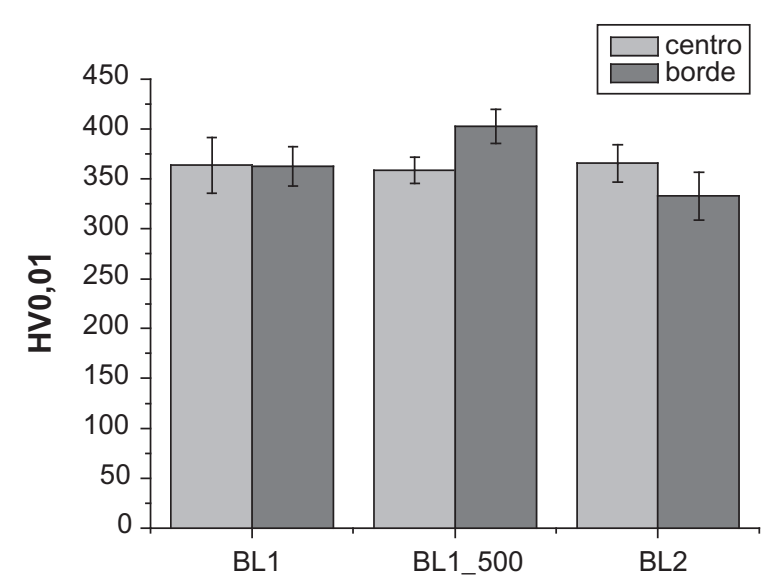

Figura 6. Microdureza determinada en el borde y centro de las probetas arenadas (BL1, BL2) y arenadas y oxidadas térmicamente (BL1_500).

Figure 6. Microhardness values at the border and centre of the sandblasted specimens $(B L 1, B L 2)$ and thermally oxidised and sandblasted specimens (BL1_500).

probeta BL1 no se ve afectada por el tratamiento de arenado, mientras que en la muestra BL2 se detecta incluso una pequeña bajada de la misma. Estos resultados contrastan con los estudios realizados en titanio ${ }^{[8]}$, en los cuales se observa que la superficie arenada su- fre tensiones de compresión pero, al contrario que en la superficie de Ti6Al4V, el valor de la dureza aumenta en las proximidades de la superficie.

Otro resultado que llama la atención es el hecho de que en la probeta que ha sido tratada y que tiene unas tensiones residuales de magnitud similar a la de las muestras pulidas, se produce un endurecimiento significativo (en este caso las barras de error no se solapan). Este efecto podría tener su origen en la disolución de oxígeno en la aleación ya que los tratamientos se han realizado al aire y el oxígeno disuelto en esta aleación produce endurecimiento ${ }^{[18]}$. Para verificar este aspecto se realizaron medidas de microdureza en probetas facilitadas por Surgival y que fueron pulidas y tratadas térmicamente en las mismas condiciones que las probetas arenadas. Los valores obtenidos para las muestras pulidas y las pulidas y oxidadas son $353 \pm 37$ y $347 \pm 18$, respectivamente. Como puede apreciarse las diferencias no son estadísticamente significativas.

Del análisis global de estos resultados se deduce la necesidad de ampliar el estudio realizando medidas con otras técnicas para profundizar en el mecanismo responsable del endurecimiento observado.

\section{CONCLUSIONES}

- El procesado de arenado no ocasiona un incremento de la dureza subsuperficial del material a pesar de la deformación tan severa experimentada; mas bien, podría hablarse de una disminución de la misma como se ha observado en el caso de la probeta BL2. Este "ablandamiento" y la presencia de grietas en la superficie son los dos factores microestructurales que podrían explicar el empeoramiento de la resistencia a la fatiga con respecto a la del material no arenado. La presencia de partículas de alúmina, actuando como concentrador de tensiones, no parece explicar dicha disminución ya que en el caso de titanio puro el arenado ocasiona un aumento significativo de la resistencia a la fluencia.

- El tratamiento térmico de oxidación incrementa la dureza subsuperficial, lo que podría ser beneficioso en relación con el comportamiento a fatiga.

\section{Agradecimientos}

Proyectos MAT2006-12948-C04-01, del Ministerio de Educación y Ciencia y Fundación Mutua Madrileña. A BESSY las facilidades para las medidas de las tensiones residuales por difracción de radiación sincrotrón bajo contrato № RII 3CT-2004- 
INFLUENCIA DEL ARENADO DE LA ALEACIÓN TI6AL4V EN LA DUREZA SUBSUPERFICIAL Y ESTADO DE TENSIONES RESIDUALES INFLUENCE OF SANDBLASTING OF TI6AL4V ALLOY ON THE SUBSURFACE HARDNESS AND RESIDUAL STRESSES STATE

506008. La Dra. M.M. agradece la financiación de su contrato Juan de la Cierva al Ministerio de Ciencia Innovación. El CIBER de Bioingeniería, Biomateriales y Nanomedicina está financiado por el ISCIII.

\section{REFERENCIAS}

[1] K. Anselme, P. Linez, M. Bigerelle, D. Le Maguer, A. Le Maguer, P. Hardouin, H.F. Hildebrand, A. Iost y J.M. Leroy, Biomaterials 21 (2000) 1.567-1.577.

[2] L. Saldaña, V. Barranco, J.L. González-Carrasco, M. Rodríguez, L. Munuera y N. Vilaboa, J. Biomed. Mater. Res. Part A, 81A (2007) 334-346.

[3] C. Aparicio, F.J. Gil, C. Fonseca, M. Barbosa y J.A. Planell, Biomaterials 24 (2003) 263-273.

[4] A. Piattelli, M. Degidi, M. Paolantonio, C. Mangano y A. Scaramo, Biomaterials 24 (2003) 4.081-4.089.

[5] A. Wennweberg, T. Albrektsson y B. Andersson, Int. J. Oral Maxillofac. Implants 11 (1996) 38-45.

[6] V.M.Goldberg, S. Stevenson, J. Feighan y D. Davy, Clin. Orthop. Relat. Res. 319 (1995) 122-129.

[7] C. Aparicio, Fj Gil, U. Thams, F. Muñoz, A. Padrós y J.A. Planell, Key Eng. Mater. 254-256 (2004) 737-740
[8] X. P. Jiang, X. Y. Wang, J. X. Li, D. Y. Li, C. S. Man, M. J. Shepard y T. Zhai, Mater. Sci Eng. A 429 (2006) 30-35.

[9] C. Leinenbach y D. Eifler, Biomaterials 27 (2006) 1200-1208

[10] F. J. Gil, J. A. Planell, A. Padrós y C. Aparicio, Dent. Mater. 23 (2007) 486-491.

[11] M. Baleani, M. Viceconti, y A. Toni, Artificial Organs 24 (2000) 296-299.

[12] S.A. Martinez, S. Sathish, M.P. Blodgett, S. Mall y S. Namjoshi, Mater. Sci. Eng. A 399 (2005) 58-63.

[13] P. Fernández-Castrillo, G. Bruno y G. GónzalezDoncel, Mater. Sci. Eng. A, 487 (2008) 26-32.

[14] C.H. Genzel, C. Stock y W. Reimers, Mater. Sci.Eng.A 372 (2004) 28-43

[15] E. Conforto, B.O. Aronsson, A. Salito, C. Crestou y D. Caillard, Mater. Sci. Eng. C 24 (2004) 611-618.

[16] J. Valenti, J. Guasch, P. Sevilla y F. J. Gil, Biomecánica 12 (2004) 24-30.

[17] I. C. Noyan y J. B. Cohen, Residual Stress Measurement by Neutron Diffraction, Ed. Springer, 1987.

[18] Z. X. Zhang, H. Dong, T. Bell, B Xu, J. Alloys Compd. 431 (2007) 93-99. 